\title{
Evaluation of the Fertility Rate of the Mouflon Semen Through Artificial Insemination in Synchronized Sheep
}

\author{
Mihai Marian BORZAN ${ }^{1 *}$, Iancu Adrian MORAR ${ }^{2}$, Emoke PALL ${ }^{2}$, Alexandru Raul POP ${ }^{2}$, Ioan PAȘCA ${ }^{1}$, \\ Adrian CÎMPEAN ${ }^{1}$, Ioan Ştefan GROZA ${ }^{2}$ \\ ${ }^{1}$ Department of Animal Productions and Food Safety, USAMV Cluj, Romania \\ ${ }^{2}$ Department of Reproduction, Obstetrics and Reproductive Pathology, USAMV Cluj, Romania \\ Corresponding author: mihai_borzan@yahoo.com
}

Bulletin UASVM Veterinary Medicine 72(1) / 2015,

Print ISSN 1843-5270; Electronic ISSN 1843-5378

DOI:10.15835/buasvmcn-vm: 11052

\begin{abstract}
The European mouflon is a wild species of sheep native in the islands of Corsica and Sardinia. The researches conducted to preserve these species play an important role in long term conservation for these animal. Experimental study was conducted with the aim to evaluate in vivo the reproductive quality of the mouflon semen, obtained by electroejaculation, using artificial insemination in sheep and evaluating the fertility rate. The sheep included in the study (36) were hormonal synchronized with progesteron and eCG (Equine Chorionic Gonadotrophin). Following synchronization, vaginal artificial insemination was performed with mouflon semen. The pregnancy diagnosys was performed by ultrasound examination at 55 and145 days after artificial insemination. The concieving rate was $36,11 \%$, the prolificacy rate was $61,53 \%$ and fecundity rate was $22,22 \%$. The data obtained after assessing the mouflon semen, collected by electrostimulation, confirmed the pretability of the semen for artificial insemination.
\end{abstract}

Keywords: artificial insemination, fertility rate, mouflon, semen.

\section{INTRODUCTION}

European mouflon (Ovis orientalis musimon) is a species of wild native ruminants from the Mediterranean Seabasin, extended into continental Europe. To the specimens of wild mouflon located in the origin area, the onset of estrus begins in October. The mouflon is not included in the list of the endangered species, but it is classified on the IUCN Red List (International Union for Conservation of Nature) as a rare species in the country of origin and therefore vulnerable (high risk of extinction is in the near future) (http:// www.iucnredlist.org, Berlinguer F,2005).

The main threats affecting the wild population of mouflons are considered to be the human activities which reduced and altered the natural habitats of this wild sheep and epidemic spread of infectious diseases, contributed to the decrease of these populations. As the result of the fragmentation in the natural environment, the wild population of mouflons is kept in small numbers, which can lead to the loss of genetic diversity (Brook BW, 2000).

Application of assisted reproductive technologies is accepted as an important tool in ensuring, on the long term, the survival of wildlife species and the restoration of lost genetic variability (Wild DE, 1997; Bainbridge DRJ, 1998; Comizzoli P, 2000; Berlinguer F, 2005).

In particular, the genetic source of cryobanks, storage and use of biological materials, from living or dead animals, can contribute to conservation of individuals and development of fundamental knowledge [Leibo S.P., 2002; Berlinguer F.2005].

The aim of this study was to evaluate, in vivo, the reproductive potential of the mouflon semen 
collected by the electroejaculation method, by using it to artificial inseminate domestic sheep.

\section{MATERIALS AND METHODS}

The research was conducted in the Andrology laboratory from the Faculty of Veterinary Medicine of USAMV Cluj-Napoca in collaboration with Turda Zoo and the production Farm S.C. "Livezile" S.R.L. Bistrița County.

The selection of the sheep included in this study was made from the sheep provided by the partners. 36 ewes were included in the study, aged between 1 and 8 years old, which were previously evaluated to be clinically healthy and a reproduction soundness examination was performed. This batch of animals was separated from the rest of the animals and a synchronization protocol was performed. Progesteron and eCG (Equine Chorionic Gonadotrophin) was used to manage the estrus cycle. The protocol used consists of Chronogest ${ }^{\circledR} \mathrm{CR}$, (Intervet ScheringPlough Animal Health), applied as vaginal sponges, each sponge contains $20 \mathrm{mg}$ cronolon and is applied one per ewe, regardless of body weight, breed or season. The sponge was left intravaginal for14 days. After the extraction of the sponge, eCG (Equine Chorionic Gonadotrophin) (Folligon) was used as 750 U.I.

The semen was collected from captive mouflons which live in Turda Zoo. They are sexually mature. Before the collection of semen the mouflons were examined to establish the health status. To capture the animals, a dart gun was used to inject the tranquilizers (Xylazine and Ketamine).

The semen was collected by electrical stimulation using a standard electroejaculator for small ruminants. Under electrical stimulation, the semen was collected in glass sterile containers dried and heated to body temperature $\left(38^{\circ} \mathrm{C}\right)$. For each collected sperm emission, the containers were changed to avoid the contamination with urine of the whole ejaculate.

The semen collected was evaluated macroscopically and microscopically to evaluate the quality of the semen. The evaluated parameters were: color, consistency, density, sperm waves, mobility, viability, concentration, contamination with urine.

All the semen samples which had important modifications were excluded from the study and the semen evaluated as good quality was used to artificially inseminate synchronized sheep. After the qualitative evaluation of the semen, the concentration of the sperm was performed. The concentration of sperm was very important in deciding the amount of extender used to obtain in the end the concentration of 300 million sperm per insemination dose. The extender used was Triladyl Bovine, a standard egg-yolk based extender for small ruminants produced by Minitube. After the two successive dilutions (first 1:1 and second to obtain the number of sperm wanted per insemination dose) the semen is rechecked to be sure that the extender didn't affect the quality of the sperm.

The prepared semen was inseminated intravaginally with a speculum at $55 \mathrm{~h}$ after the removal of the sponges. Artificial insemination was performed with $300 \mu \mathrm{l}$ of semen with a concentration of more than 300 million sperm per insemination dose. The restraining of sheep was done in an insemination stand.

The pregnancy diagnosis of inseminated sheep was established by transabdominal ultrasound at 55 days after artificial insemination.

The formulas for fertility, prolificacy and fecundity are (Ptaszynska M, 2009):

$$
\begin{aligned}
& \text { fertility }=\frac{\text { number of pregnant sheep }}{\text { number of inseminated sheep }} X 100 \\
& \text { prolificacy }=\frac{\text { number of delivered lambs (dead and alive) }}{\text { number of pregnant ewes }} X 100 \\
& \text { fecundity }=\frac{\text { number of delivered lambs (dead and alive) }}{\text { number of inseminated sheep }} X 100
\end{aligned}
$$

\section{RESULTS AND DISCUSSION}

The clinical examination revealed that all the animals included in the study were healthy and no reproductive pathology was found.

In our research, the vaginal examination performed with an vaginal speculum, allowed us to aperciate the cervical status of each inseminated ewe. All the inseminated ewes had the signs of oestrus at the time of the vaginal insemination (Tab.1).

At 55 days after insemination, an ultrasound examination was performed to establish the gestation status. We found that 13 out of 36 ewes were pregnant following the artificial insemination performed with fresh extended mouflon semen obtained by electrical stimulation, 
Tab.1. Results of vaginal examination in ewes at the time of the artificial insemination

\begin{tabular}{|c|c|c|c|}
\hline \multirow{2}{*}{\multicolumn{2}{|c|}{ Vaginal examination }} & \multicolumn{2}{|c|}{ Total } \\
\hline & & 36 & $\%$ \\
\hline \multirow{3}{*}{ Cervix } & Hyperemia & 26 & 72.22 \\
\hline & Hyperemia and opened & 10 & 27.78 \\
\hline & Without hyperemia & 0 & 0 \\
\hline \multirow{2}{*}{\multicolumn{2}{|c|}{ Vaginal examination }} & \multicolumn{2}{|c|}{ Total } \\
\hline & & 36 & $\%$ \\
\hline \multirow{2}{*}{ Vaginal collection / } & Present & 31 & 86.11 \\
\hline & Absent & 5 & 13.89 \\
\hline
\end{tabular}

which represented $36,11 \%$ of the inseminated batch.

A second ultrasound examination was performed at 145 days after artificial insemination. At 145 days, from 13 pregnant females, only 5 were still pregnant (13.89\%), the others aborted in this period. Between 153 and 158 days after artificial insemination the pregnant ewes lambed naturally 8 mixed lambs (mouflon x ewe) - 7 alive and 1 dead. Two ewes lambed two and three lambs (Fig.1, 2).

For fresh semen, the range for pregnancy rate reported can vary between $40-65 \%$ in intravaginal insemination, 50-70\% in intracervical insemination and $60-90 \%$ in laparoscopic insemination (Ptaszynska M, 2009).

In our research, after statistical analysis the following results for artificial insemination of the ewes:

$\begin{array}{cc}\text { Fertility } & 36.11 \% \\ \text { Prolificacy } & 61.53 \% \\ \text { Fecundity } & 22.22 \%\end{array}$

It is well known that the reproductive behavior of the mouflon is similar to that of sheep, (Valdez R et al., 1978, Deligiannis C et al., 2005, Hosseini $\mathrm{SM}, 2009)$. This is the main reason why we choose the domestic sheep to test the reproductive potential of the mouflon semen collected by electrostimulation. Another reason was the availability of domestic sheep compared with wild sheep and the better response of the reproduction system to synchronization of the domestic sheep.

The use of assisted reproductive biotechnologies for the endangered mammalian species is limited by some factors: the success in producing new offspring depends on the understanding of reproductive physiology of each species, and in wild animals it is not fully understood; the captivity and the restricted biological material available makes very difficult to be scientifically approached, so the development of these reproductive biotechnologies depends on a multidisciplinary research (Comizzoli P, 2000).

Most of the reproductive biotechnologies applied to wild species come from the model found in a similar domestic species. The wild species of animals are more sensitive to stress compared with the domestic species, and that is the reason why the effective work of the researcher for a long period of time is very hard to be achieved, considering the high risks involving the animal's welfare (Comizzoli P et al., 2000).

Another problem in using the reproductive biotechnologies for wild animals is the logistic involved in moving the gear and materials needed for scientific research, from the laboratory, to the field, in their environment. The use of the conservation programs for wild endangered species is less used compared to the rare domestic species (Comizzoli $\mathrm{P}, 2000$ ).

The success of artificial insemination depends on three important factors: sperm can survive outside of the male genital tract; they can be introduced in the female genital tract with acceptable conceiving rates; the optimal time for insemination. If all these are achieved, the artificial insemination will be successful. In some species, this considerations are easily achieved, and artificial insemination is very well used on a large scale, but there are other species were the artificial insemination is rarely performed because this considerations are not well covered (Noakes DE, 2009).

For small ruminants, the success of artificial insemination depends on the reproductive mana- 

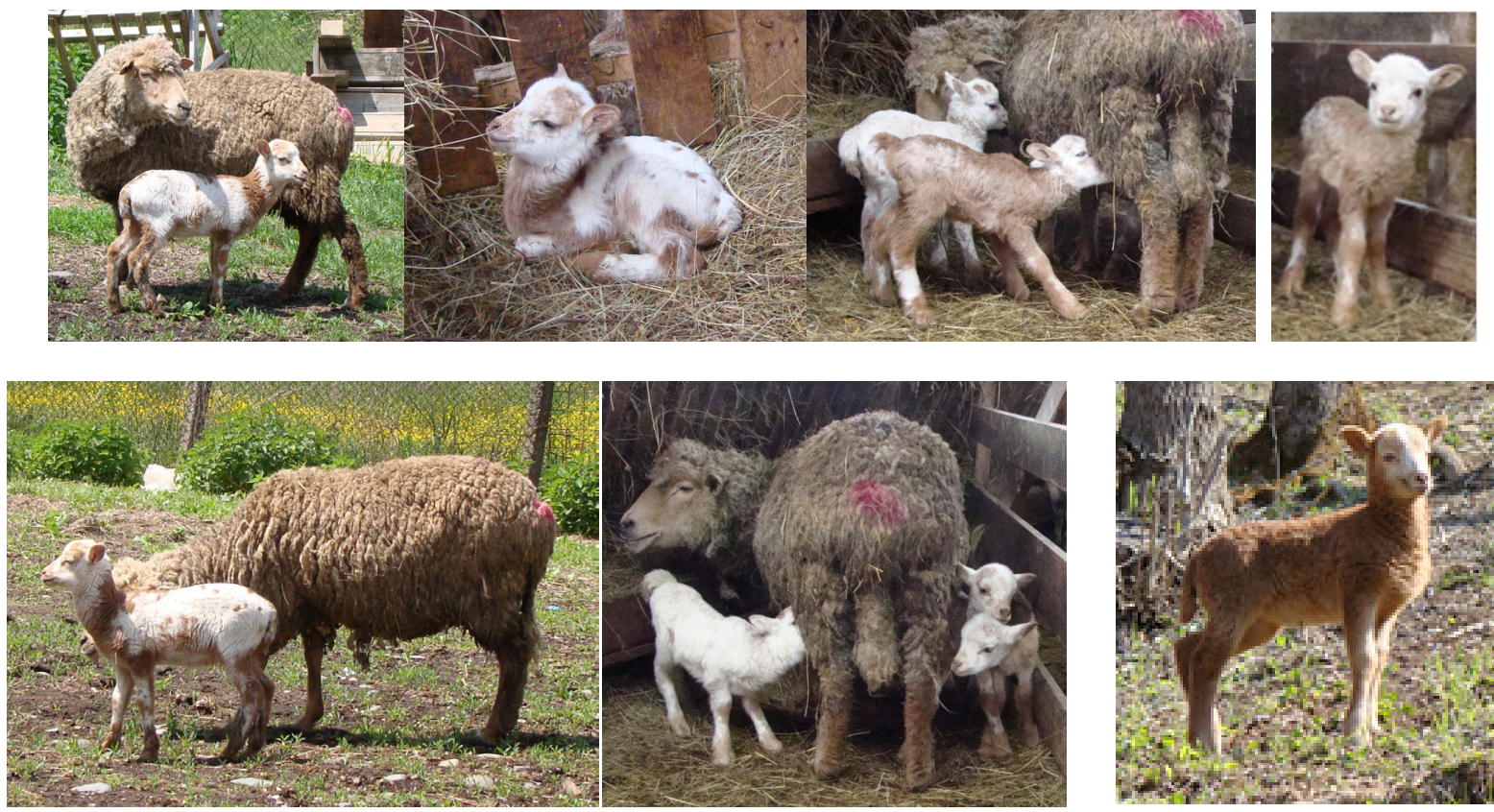

Fig. 1. The crossed lambs resulted out of mouflon $x$ sheep

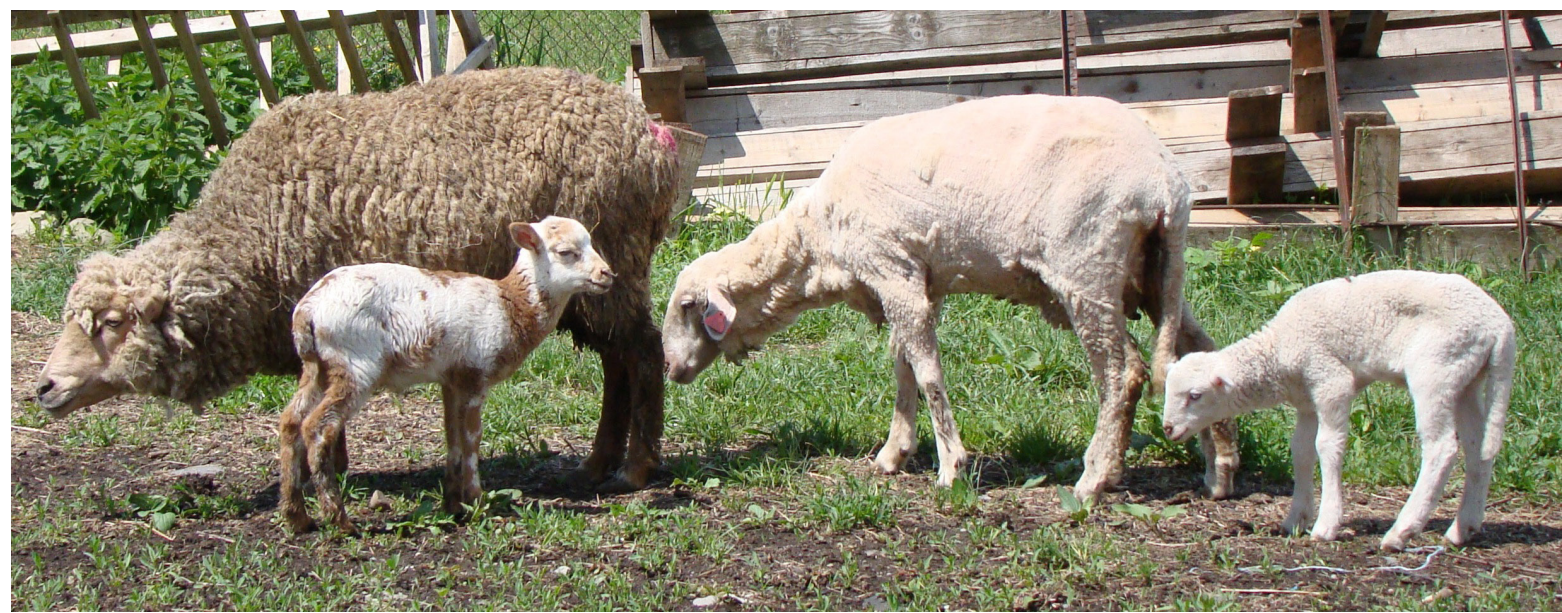

Fig. 2. Lamb resulted out of artificial insemination with mouflon (left) and purebreed lamb(right)

gement of the females, but also on the number, concentration, progressive motility of sperm, time of insemination and the way of insemination. Although the morphocytometric analysis of sperm is considered to be essential for the quality evaluation of sperm used in artificial insemination, the pregnancy rate is more accurate to assess the success of the method (Groza IŞ, 2006).

For the intravaginal insemination of extended semen, the minimal number of sperm/ insemination doses must be at least 300 millions sperm to obtain an average pregnancy rate of $50 \%$ (Ptaszynska M, 2009).

\section{CONCLUSION}

The semen collected by electrostimulation was suitable to be extended and used for artificial insemination.The results showed that mouflon semen obtained by electrostimulation was suitable for artificial insemination, fact prooved by the pregnancy rate obtained.

The synchronization method used in this study prooved to be succesful in artificial insemination with mouflon semen.

Following the artificial insemination of the ewes included in the research, with mouflon semen the fertility rate was $36,11 \%$, prolificacy rate was $61,53 \%$, and fecundity rate was $22,22 \%$. 
The fertility percent obtained after artificial insemination proved the suitability of the mouflon's semen collected by electrostimulation to be used in artificial insemination.

\section{REFERENCES}

1. Bainbridge DRJ, Jabbour HN (1998). Potential of assisted breeding techniques for the conservation of endangered mammalian species in captivity: A Review. Vet Rec 143:159-168.

2. Berlinguer F, Giovanni GL, Bogliolo L, Bebbere D, Succu S, Rosati I, Ledda S, Naitana S (2005). In vivo and in vitro fertilizing capacity of cryopreserved European mouflon (Ovis gmelini musimon) spermatozoa used to restore genetically rare and isolated populations. Theriogenology 63:902-911.

3. Brook BW, O'Grady JJ, Chapman AP, Burgman MA, Akcakaya HR, Frankham R (2000). Predictive accuracy of population viability analysis in conservation biology. Nature 404:385-7.

4. Colenbrander B, Gaella BM, Stout TAE (2003). The predictive value of semen analysis in the evaluation of stallion fertility. Reprod Domest Anim 38:305-311.

5. Comizzoli P, Mermillod P, Mauget R (2000). Reproductive biotechnologies for endangered mammalian species. Reprod Nutr Dev 40:493-504. doi:10.1051/rnd:2000113

6. 6.Deligiannis C, Valasi I, Rekkas CA, Goulas P, Theodosiadou E, Lainas T, Amiridis GS (2005). Synchronization of ovulation and fixed time intrauterine insemination in ewes. Reprod Domest Anim 40(1):6-10.

7. 7.Groza IS (2006). Ginecologie, andrologie și obstetrică veterinară - compendiu. Ed. Academiei Române, București.

8. Hosseini SM, Fazilati M, Moulavi F, Foruzanfar M, Hajian M, Abedi P, Nasiri N, Kaveh AK, Shahverdi AH, Hemami MR, Nasr-Esfahani MH (2009). Reproductive potential of domestic Ovis aries for preservation of threatened Ovis orientalis isphahanica: in vitro and in vivo studies. Eur J Wildl Res 55:239-246. DOI 10.1007/s10344-008-0242-3.

9. Leibo SP, Songsasen N (2002). Cryopreservation of gametes and embryos of non-domestic species. Theriogenology 57:303-326.

10. Noakes DE, Parkinson TJ, England GCW (2009). Veterinary reproduction and Obstetrics. Ninth Edition, Saunders Elsevier, 708-709, 716.

11. Ptaszynska M editor (2009) $.10^{\text {th }}$ edition of Compendium of animal reproduction, Publisher Intervet International bv, ISBN 90-801886-6-2.

12. Valdez R, Nadler CF, Bunch TD (1978). Evolution of wild sheep in Iran. Evolution, 32: 56-72.

13. Wildt DE, Rall WF, Critser JK, Monfort SL, Seal US (1997). Genome resource banks. Living collections for biodiversity conservation. Bioscience 47:689-698.

14. http://www.iucnredlist.org/ 